\title{
La vicinanza solidale in contesti di vulnerabilità familiare. La partecipazione nella comunità come strada per ricostruire $i$ tessuti sociali
}

\author{
Sara Serbati, Andrea Petrella ${ }^{2,3}$
}

\begin{abstract}
La vulnerabilità familiare è una condizione sociale ed educativa caratterizzata da un'elevata complessità relazionale, che può avere gravi conseguenze per il soddisfacimento dei bisogni di crescita di un bambino. La vicinanza solidale, intesa come forma di solidarietà tra famiglie finalizzata a sostenere un nucleo familiare attraverso il supporto di altri nuclei o di singole persone, è una delle risorse che il Programma P.I.P.P.I. (Programma di Intervento Per la Prevenzione dell'Istituzionalizzazione) propone di attivare per fronteggiare situazioni di vulnerabilità ed esclusione sociale. L'articolo presenta un'esperienza di approfondimento realizzata a Sondrio all'interno di P.I.P.P.I., nella quale gli operatori, accompagnati dai ricercatori, hanno promosso un dialogo con le famiglie. Queste occasioni di confronto hanno generato nuovi e inaspettati percorsi che interrogano la vicinanza solidale quale processo educativo che risponde ai bisogni di bambini e famiglie, e che valorizza la partecipazione nella comunità come strada per ricostruire i tessuti sociali delle persone.
\end{abstract}

Parole chiave: vicinanza solidale, famiglie vulnerabili, solidarietà, intersoggettività, comunità.

\begin{abstract}
Family vulnerability is a particular social situation, characterised by high levels of relational complexity, with serious consequences for the fulfilment of the developmental needs of a child. In the framework of the P.I.P.P.I. Programme (Programme of Interven-
\end{abstract}

${ }^{1}$ Ricercatrice in Pedagogia generale e sociale presso il Dipartimento di Filosofia, Sociologia, Pedagogia e Psicologia applicata dell'Università degli Studi di Padova.

${ }^{2}$ Assegnista di ricerca presso il Dipartimento di Filosofia, Sociologia, Pedagogia e Psicologia applicata dell'Università degli Studi di Padova.

${ }^{3}$ L'articolo è frutto del lavoro congiunto degli Autori. Tuttavia è possibile attribuire la responsabilità nella redazione come di seguito: Introduzione e parr. 2, 3, 4, 6 a Sara Serbati; par. 5 ad Andrea Petrella; i Ringraziamenti sono da intendersi a cura di entrambi gli Autori, N.d.Aa. 
tion for the Prevention of Institutionalisation), social support is intended as a form of solidarity between families that aims to help a family through the support of another family, or individuals. In order to face vulnerability and social exclusion, P.I.P.P.I. promotes the mobilisation of these resources. The paper is therefore focused on a case study based in Sondrio (Italy), carried out within the P.I.P.P.I. Programme. The professionals together with the researchers tested their understanding, also promoting dialogue-meetings with families. These dialogical and interactive contexts generated new and unexpected interpretations of this phenomenon, framing it into an educational process that meets the needs of children and families, and promoting community participation as a way to reconstruct local social networks as well.

Keywords: social support, vulnerable families, solidarity, intersubjectivity, community.

\section{Introduzione}

L'articolo intende presentare un'esperienza di co-ricerca riguardante il tema della vicinanza solidale. L'esperienza si è compiuta all'interno del Programma P.I.P.P.I. (Programma di Intervento Per la Prevenzione dell'Istituzionalizzazione) diretto dall'Università di Padova e dal Ministero del Lavoro e delle Politiche Sociali, che ne è anche l'ente finanziatore (Milani et al., 2015). Il Programma, avviato nel 2011, prevede ogni anno la selezione di 50-60 ambiti territoriali ad opera delle Regioni, per la realizzazione di un biennio di implementazione con dieci famiglie con figli tra 0 e 11 anni. P.I.P.P.I. si concentra sul sostegno a bambini e famiglie attraverso interventi multiprofessionali, olistici e basati sulla resilienza, al fine di ridurre la povertà educativa e la negligenza familiare (Lacharité, 2014; Id. et al., 2006) e mira a rispondere ai bisogni dei bambini mediante un'azione collettiva (Serbati, 2016), di promozione della resilienza personale e familiare, e delle capacità della comunità (Chaskin et al., 2001).

L'esperienza del Programma P.I.P.P.I. è divenuta soft law il 21 dicembre 2017, quando la Conferenza unificata Stato-Regioni ha approvato le Linee di indirizzo nazionali intitolate L'intervento con bambini e famiglie in situazione di vulnerabilità. Promozione della genitorialità positiva. Si è trattato del frutto di un lavoro collegiale e pluriennale, realizzato in seno a un tavolo interistituzionale nazionale, promosso dal Ministero del Lavoro e delle Politiche Sociali, composto da rappresentanti del Ministero stesso, della Conferenza dei Presidenti delle Regioni e delle Province autonome, e del gruppo scientifico dell'Università di Padova responsabile del Programma P.I.P.P.I. Da allora, P.I.P.P.I. è divenuto azione per l'implementazione delle Linee di indirizzo e figura stabilmente nel Fon- 
do Nazionale per le Politiche Sociali. Ad oggi il Programma ha coinvolto tutte le 20 Regioni italiane, per 238 Ambiti territoriali (113 dei quali per più edizioni), circa 4.000 famiglie e una comunità di pratiche e di ricerca formata da più di 8.000 operatori dei servizi sociali, educativi, sociosanitari e della scuola.

Nel biennio 2017-2018 è stato avviato il livello avanzato di P.I.P.P.I., riservato a chi tra gli Ambiti territoriali ha già completato almeno un biennio intero di sperimentazione, e che dunque manifesta esigenze di implementazione diversificate rispetto agli Ambiti che si apprestano a sperimentare il Programma per la prima volta.

Molte di queste esigenze sono relative a garantire trasferibilità all'expertise acquisita, avviando percorsi formativi e post-formativi volti a integrare gli elementi chiave del metodo e dell'organizzazione di P.I.P.P.I. negli assetti tecnici, organizzativi e istituzionali, in maniera stabile (LabRIEF, 2019a, p. 1).

L'attivazione del livello avanzato persegue la finalità di costruire insieme agli Ambiti territoriali quelle condizioni organizzative che garantiranno a ciascun territorio di radicare, trasformare, adattare le proposte di P.I.P.P.I. realizzando una progressiva autonomia e capitalizzando le competenze acquisite nella/e prima/e implementazione/i. Attraverso l'introduzione di un nuovo soggetto, il Laboratorio Territoriale (LabT), gli ambiti sperimentano «contesti di co-ricerca tra professionisti e universitari finalizzati alla produzione di innovazione sociale» (Di Masi et al., 2019, p. 96), mettendo al centro della riflessione «la relazione tra gli attori come luogo in cui costruire conoscenza attraverso percorsi di indagine condivisi e partecipati, orientati a produrre un impatto sulle pratiche» (Ibidem).

L'Ambito territoriale di Sondrio (22 Comuni per 55.746 abitanti) dal 2014 partecipa al Programma P.I.P.P.I. e in occasione della sesta (20172018) e settima (2018-2019) implementazione decide di aderire al livello avanzato, intraprendendo un processo di co-ricerca e di costruzione di innovazione sociale nel proprio territorio. Il Laboratorio Territoriale di Sondrio, composto inizialmente dal referente dei servizi sociali, da operatori esperti del Programma P.I.P.P.I. e dai ricercatori dell'Università di Padova, si arricchisce nel tempo di ulteriori partecipazioni provenienti dal mondo della scuola, del Terzo settore e del volontariato. Negli anni 2018-2019 il LabT svolge analisi e riflessioni a partire dai dati disponibili sul Programma P.I.P.P.I. in riferimento alle implementazioni precedenti e decide di elaborare un percorso di co-ricerca sul tema della vicinanza 
solidale, come area specifica del lavoro con le famiglie su cui progettare un percorso di innovazione.

\section{La vicinanza solidale in P.I.P.P.I}

La vulnerabilità, e con essa la negligenza familiare, sono considerate in P.I.P.P.I. un problema sociale complesso, cui rispondere con l'adozione di azioni collettive che si sviluppano attraverso quattro dispositivi d'intervento, ciascuno dei quali ha trovato definizione all'interno delle summenzionate Linee di indirizzo (Ministero del Lavoro e delle Politiche Sociali, 2017). Essi sono: il servizio di educativa domiciliare, il gruppo per $i$ genitori, il partenariato scuola-famiglia-servizi, la vicinanza solidale. È possibile considerare come i quattro dispositivi propongano interventi che supportano le famiglie nelle relazioni sia all'interno del nucleo, sia all'esterno, con un'attenzione a quelle relazioni che ne costruiscono l'intorno, come le relazioni con la scuola, o le relazioni con altri genitori.

Il presente articolo si focalizza in particolare sull'ultimo dei dispositivi in elenco, la vicinanza solidale, inteso come opportunità per le famiglie che vivono particolari situazioni di vulnerabilità e negligenza di poter trovare e costruire nel proprio ambiente di vita occasioni per essere aiutati nel dare risposte ai bisogni di sviluppo dei bambini. Di seguito la definizione che se ne propone all'interno delle Linee di Indirizzo:

la Vicinanza Solidale rappresenta una forma di solidarietà tra famiglie che ha come finalità quella di sostenere un nucleo familiare attraverso la solidarietà di un altro nucleo o di singole persone in una logica di affiancamento e di condivisione delle risorse e delle opportunità (ivi, p. 71).

La proposta di P.I.P.P.I. e delle Linee di indirizzo poggia su importanti esperienze sia nazionali che internazionali (Milani, 2006). Alcune di esse ormai vantano numerosi anni di esperienza: sono i parents visiteurs del Québec (Durand et al., 1989) e i family belpers negli USA (Lines, 1987). Altre, sono più recenti, ma non per questo meno importanti: la Rete di Famiglie Aperte del Coordinamento nazionale delle comunità di accoglienza (Tuggia, 2006), l'affiancamento familiare promosso dalla Fondazione "Paideia" (Maurizio et al., 2015), o l'esperienza delle famiglie in rete che ha avuto inizio ad Asolo (Tv) e si sta rapidamente diffondendo (Borsellino, Belotti, 2010). 
Tutte queste esperienze raccontano dell'efficacia di un approccio che fa riferimento all'aiuto che può derivare a una famiglia nel compito di rispondere ai bisogni dei bambini, da parte di altre persone o di un'altra famiglia. Queste esperienze vengono mutuate e ulteriormente elaborate prima dal Progetto P.I.P.P.I., poi dalle Linee di indirizzo, con il riferimento al costruirsi di una relazione tra due soggetti, uno dei quali si trova nella condizione di richiedere aiuto e supporto, che riceve dall'altro in maniera informale e non professionale (Folgheraiter, Cappelletti, 2011), privilegiando «la dimensione informale dell'intervento e la creazione e/o potenziamento di reti sociali» (Ministero del Lavoro e delle Politiche Sociali, 2017, p. 71) intorno alla famiglia, «nella vita quotidiana, a partire da bisogni circoscritti e concreti, individuati insieme alla famiglia» (Ibidem).

Nei processi decisionali alla base della relazione d'aiuto, la partecipazione della famiglia che riceve l'aiuto, nonché della famiglia che lo dona, appare dunque di fondamentale importanza: «richiede un tempo e uno spazio in cui, operatori e famiglia, cercano insieme di identificare il bisogno che può essere soddisfatto con la vicinanza solidale, riconoscendo alla famiglia un ruolo attivo e da protagonista in questo percorso» (Tuggia, 2017, p. 83). Le Linee di indirizzo sulla vulnerabilità familiare prevedono tre aree rispetto alle quali procedere con un attento dialogo relativamente ai bisogni cui dare risposta tramite la vicinanza solidale: nei confronti delle risposte da offrire ai bisogni del bambino, nell'accompagnamento dei genitori rispetto alle loro risposte ai bisogni del bambino, nella costruzione di reti e relazioni sociali intorno a quest'ultimo e alla sua famiglia.

Uno dei frequenti limiti incontrati in questi anni di sperimentazione è rappresentato dal momento dell'avvio del dispositivo, in cui le Linee di indirizzo raccomandano di «garantire che le persone disponibili alla vicinanza solidale siano individuate il più vicino possibile all'ambiente di vita della famiglia» (Ministero del Lavoro e delle Politiche Sociali, 2017, p. 72) prevedendo una gradualità:

prima di tutto all'interno della sua rete familiare, amicale e sociale, tramite un'attenta mappatura delle risorse, valorizzando anche le famiglie stesse che hanno ricevuto il medesimo aiuto in precedenza;[...] qualora dall'esplorazione della rete familiare, amicale e sociale non emergessero persone disponibili, si rivolgono ad associazioni e reti di famiglie presenti nel territorio per individuare persone che siano disponibili a svolgere funzioni di vicinanza solidale, senza che questo comporti necessariamente la stipula di convenzioni a carattere oneroso (Ibidem). 


\section{Obiettivi e domanda di ricerca}

All'interno di tutte le implementazioni di P.I.P.P.I. la vicinanza solidale ha dimostrato la propria efficacia relativamente ai risultati raggiunti (Labrief, 2019b; Serbati, 2016). Tale dato conferma le tesi presenti in letteratura sul rapporto positivo delle esperienze di vicinanza solidale, che creano condizioni facilitanti per i genitori nel rispondere ai bisogni dei figli, e quindi tra la presenza di relazioni sociali e lo sviluppo cognitivo, emotivo e sociale del bambino (Hostinar et al., 2014; Taylor, 2011).

L'importanza delle azioni di rafforzamento della rete di sostegno alle famiglie è ben presente nel pensiero degli operatori sociali. Il concetto di community care, con la priorità attribuita all'esigenza delle persone che accedono ai servizi, di vivere, crescere, e sperimentare cambiamenti all'interno della comunità locale di appartenenza, è ormai entrato nei presupposti operativi dei servizi (Raineri, 2013). Tuttavia, i dati che riguardano le percentuali di attivazione di questo dispositivo testimoniano la fatica nella realizzazione di tale intervento. In tutte le implementazioni la vicinanza solidale è sempre il dispositivo con la minore percentuale di attivazione, intorno al 50\% del totale delle famiglie. Sembra dunque esserci uno scollamento tra ciò che gli operatori affermano e sanno e gli interventi effettivamente realizzati.

Proprio questo scollamento è stato riscontrato anche dal LabT di Sondrio, sia formalmente nella lettura dei dati delle precedenti implementazioni di P.I.P.P.I., che riportavano lo scarso utilizzo del dispositivo, sia tramite azioni di mappatura delle risorse del territorio (volontariato, associazioni ecc.) e momenti di approfondimento tra attori interni ed esterni al LabT stesso: operatori sociali, volontari, amministratori locali, dirigenti di servizi, referenti di cooperative, medici. Con l'ausilio di schede di rilevazione e di strumenti come il World Café (processo di conversazione strutturato per la condivisione della conoscenza) (Brown, Isaacs, 2005), l'Ambito si è infatti interrogato sul tema del fronteggiamento delle vulnerabilità attraverso l'attivazione di reti informali attorno alle famiglie. Se da una parte emerge e si conferma il valore delle risorse di supporto, dall'altra vengono sottolineate le difficoltà nel facilitare, valorizzare e promuovere tali relazioni a beneficio delle famiglie e dell'intera collettività. Di qui, la decisione di soffermarsi sull'analisi (assessment) del proprio contesto in un processo in cui il dato raccolto a partire dalle esperienze di vicinanza solidale è stato utilizzato come base per alimentare le analisi e il confronto interno al LabT stesso, con i ricercatori e con la cittadinanza. Le diverse letture hanno aperto interrogativi e desideri 
di approfondimento, che si sono tradotti in nuovi percorsi per acquisire ulteriori conoscenze.

Le azioni di co-ricerca realizzate nel territorio di Sondrio all'interno del percorso avanzato della settima implementazione di P.I.P.P.I. hanno dato l'opportunità di raccogliere e mettere a confronto e in dialogo le voci di chi il supporto sociale lo ha vissuto ricevendolo, oppure fornendolo in maniera informale (volontari, famiglie accoglienti ecc.), o per professione (operatori sociali ed educativi, insegnanti), indagando e mettendo alla prova la definizione fornita dalle Linee di indirizzo e interrogandosi sulle condizioni che consentono di realizzare l'incontro fra chi l'aiuto lo cerca e chi lo mette in campo.

\section{Metodologia}

Il livello avanzato del Programma P.I.P.P.I. attribuisce centralità al «ruolo della ricerca nell'innovazione sociale» (Di Masi et al., 2019, p. 96), basandosi su una epistemologia costruttivista dove la realtà non è unica e oggettiva, ma multipla, frutto di una co-costruzione da scoprire attraverso una comprensione dei significati attribuiti a essa insieme alle persone che la vivono. «Intraprendere processi di innovazione sociale non richiede soltanto di situarsi entro una visione collaborativa della ricerca e delle sue tecniche, ma di pensare la ricerca come processo partecipato di apprendimento che produce un impatto concreto sulle pratiche» (Ibidem). L'epistemologia costruttivista è dunque ricompresa in una visione pragmatica della realtà, che vede la conoscenza come un processo attivo di intervento su di essa (Dewey, 1938; Peirce, 1992). Centralità è data alla partecipazione degli attori (nel nostro caso ricercatori, operatori e genitori) alla costruzione di una conoscenza della realtà che non è separabile dalla funzione di consentire un'efficace azione su di essa e di emancipazione delle pratiche (Bondioli, Ferrari, 2004; Dewey, 1938; Guba, Lincoln, 1989).

Nel definire i metodi delle azioni di co-ricerca si è fatto riferimento ad alcune esperienze che hanno posto al centro il confronto dialogico tra operatori dei servizi sociali e cittadini, in cui questi ultimi, supportati dai ricercatori, hanno trovato luoghi, tempi e spazi per elaborare il proprio punto di vista rispetto a un determinato tema, per poi esporlo come materiale di discussione con gli operatori dei servizi sociali (Allegri et al., 2020; Natland, 2015). Sulla scorta di queste esperienze, nell'autunno 2019 sono stati realizzati tre laboratori riflessivi in cui han- 
no partecipato complessivamente 16 operatori e 19 persone (definiti natural helpers, v. Tab. 1), che nell'ultimo anno in corso avevano vissuto esperienze di vicinanza solidale a fianco di famiglie e bambini, che per la loro situazione di vulnerabilità familiare accedono all'accompagnamento dei servizi sociali.

L'aiuto offerto era avvenuto in forma individuale o collettiva, ovvero all'interno di associazioni, parrocchie, doposcuola, oratori, centri di aggregazione ecc.). I laboratori sono stati costruiti con i membri del Laboratorio Territoriale di Sondrio e pensati ad boc come setting di ricerca assimilabile a un focus group. L'obiettivo che il Laboratorio Territoriale insieme ai ricercatori si è proposto di perseguire tramite i laboratori riflessivi ha riguardato la valorizzazione della voce delle famiglie e dei $n a-$ tural helpers per conoscere e approfondire le opportunità e le esperienze di vicinanza solidale già presenti e avviare un percorso di riflessione comune su come promuovere e diffondere l'attivazione di tali esperienze per le famiglie più vulnerabili.

Tab. 1 - Partecipanti ai tre laboratori riflessivi

\begin{tabular}{ll}
\hline$\#$ & Abbreviazione \\
\hline \multicolumn{2}{l}{ Laboratorio riflessivo 1 } \\
Partecipante 1 & Lab1, F1 \\
Partecipante 2 & Lab1, F2 \\
Partecipante 3 & Lab1, F3 \\
Partecipante 4 & Lab1, F4 \\
Partecipante 5 & Lab1, F5 \\
Partecipante 6 & Lab1, F6 \\
Partecipante 7 & Lab1, F7 \\
Operatore 1 & Assistente sociale, Comune \\
Operatore 2 & Assistente sociale, Comune \\
Operatore 3 & Assistente sociale, Comune \\
Operatore 4 & Psicologa, ATS \\
Operatore 5 & Educatrice nido \\
Operatore 6 & Educatrice scuola infanzia \\
\hline
\end{tabular}




\begin{tabular}{ll}
\hline$\#$ & Abbreviazione \\
\hline Laboratorio riflessivo 2 \\
Partecipante 1 & Lab2, F1 \\
Partecipante 2 & Lab2, F2 \\
Partecipante 3 & Lab2, F3 \\
Partecipante 4 & Lab2, F4 \\
Partecipante 5 & Lab2, F5 \\
Partecipante 6 & Lab2, F6 \\
Partecipante 7 & Lab2, F7 \\
Operatore 1 & Assistente sociale, Comune \\
Operatore 2 & Assistente sociale, Comune \\
Operatore 3 & Educatrice domiciliare \\
Operatore 4 & Educatore domiciliare \\
Operatore 5 & Psicologo, ATS \\
Laboratorio riflessivo 3 \\
Partecipante 1 & Lab3, F1 \\
Partecipante 2 & Lab3, F2 \\
Partecipante 3 & Lab3, F3 \\
Partecipante 4 & Lab3, F4 \\
Partecipante 5 & Lab3, F5 \\
Operatore 1 & Assistente sociale, Comune \\
Operatore 2 & Referente cooperativa sociale \\
Operatore 3 & Insegnante \\
Operatore 4 & Educatrice domiciliare \\
Operatore 5 & Psicologa, ATS \\
\hline
\end{tabular}

La conduzione dei laboratori riflessivi ha previsto due fasi.

Fase 1. Nelle prime due ore i ricercatori hanno accompagnato i partecipanti definiti natural helpers nel dare forma e contenuto alla loro idea di vicinanza solidale attraverso una discussione che ha affrontato una duplice questione:

- quali sono le persone e i luoghi in cui come genitore chiedi/cerchi aiuto? 
- [in seguito alla lettura di due brevi storie rappresentanti situazioni di vulnerabilità familiare] quali sono gli aiuti che potresti offrire? quali sono gli aiuti che secondo te sarebbero necessari?

I ricercatori hanno accompagnato i natural helpers nell'organizzazione dei contenuti emersi nella discussione, in modo tale da poterli presentare agli operatori (educatori, assistenti sociali, psicologi, insegnanti) durante la seconda fase del laboratorio.

Fase 2. Nella successiva ora, i partecipanti-natural belpers hanno esposto quanto discusso e condiviso in gruppo agli operatori, soffermandosi tanto sulle risorse sociali informali esistenti, quanto su quelle ancora da attivare e sulle connessioni fra loro. Nell'idea di utilizzare i dati raccolti come stimoli generativi di riflessione (Bove, 2009), è stata svolta una prima analisi dei dati che è stata restituita e discussa con tutti i partecipanti ai tre laboratori riflessivi (operatori e natural helpers). In questo modo è stato possibile concordare e verificare i contenuti delineati durante i tre laboratori riflessivi.

Al fine di disporre di un ulteriore approfondimento sull'esperienza della vicinanza solidale nel territorio, alcune settimane dopo la realizzazione dei laboratori riflessivi sono state invitate dai servizi, e intervistate individualmente da due ricercatori, sette persone coinvolte a vario titolo in relazioni di supporto informale. Quattro di esse avevano offerto aiuto a famiglie, bambini, coppie o singoli individui e tre avevano ricevuto uno o più aiuti in momenti particolari della loro vita.

Tab. 2 - Interviste in profondità condotte dai ricercatori

\begin{tabular}{lll}
\hline$\#$ & Abbreviazione & Offre/Riceve aiuto \\
\hline Intervista 1 & I1 & Offre aiuto \\
Intervista 2 & I2 & Riceve aiuto \\
Intervista 3 & I3 & Offre aiuto \\
Intervista 4 & I4 & Riceve aiuto \\
Intervista 5 & I5 & Offre aiuto \\
Intervista 6 & I6 & Riceve aiuto \\
Intervista 7 & I7 & Offre aiuto \\
\hline
\end{tabular}

Le conversazioni dei laboratori riflessivi, dell'incontro di restituzione e delle interviste sono state audio-registrate, trascritte e sottoposte ad 
analisi tematica dei contenuti. I risultati delle analisi hanno portato alla redazione di un rapporto di ricerca che è stato condiviso con i componenti del LabT.

\section{Risultati}

I contenuti emersi nei laboratori riflessivi sono stati suddivisi e riordinati per tematiche. I partecipanti, infatti, sollecitati dalle domande dei ricercatori e stimolati dalla lettura di alcune storie reali, riportanti le dinamiche di alcuni nuclei familiari in situazione di vulnerabilità familiare, hanno idealmente tratteggiato una serie di cerchie sociali all'interno delle quali rivolgersi per poter trovare risposta ai bisogni propri e/o dei figli, che si creano nel susseguirsi della vita familiare. Il concetto di cerchia sociale che qui richiamiamo è quello definito da Georg Simmel ([1910], 1983), secondo cui

la società è solo il nome con cui si indica una cerchia di individui, legati l'un l'altro da varie forme di reciprocità [...], tali da influenzarsi a vicenda e comportarsi secondo la determinazione che ricevono. [...] la società prescrive che gli individui siano legati da una determinazione e da un flusso reciproci. Pertanto, essa è qualcosa di funzionale cui gli individui si rapportano in senso attivo e passivo e, dato questo suo carattere, più che di società, bisognerebbe parlare di associazione (trad. it. in Iannone, 2007, p. 55).

In questa sede, quindi, per cerchia sociale intendiamo un costrutto di attori sociali che interagiscono fra loro in forma individuale o collettiva (gruppi formali e non, istituzioni, luoghi) e le relazioni che sussistono fra loro, variabili per intensità, modalità, direzione, reciprocità. Sulla base di quanto emerso dai laboratori riflessivi e dalle interviste individuali le cerchie ricalcano, in una certa misura, quelle evidenziate da studi precedenti relativi al tema della vicinanza solidale per le famiglie vulnerabili (Milani et al., 2015; Serbati, 2016; Tuggia, 2017).

I dialoghi hanno restituito non solo un'immagine di ciò che è avvenuto nel momento in cui i partecipanti hanno avuto necessità di un aiuto, ma anche di ciò che, potenzialmente, potrebbe avvenire a fronte di particolari, eventuali esigenze. La particolarità di questi confronti, quindi, è stata quella di raccogliere racconti basati su esperienze reali, insieme a considerazioni e riflessioni suscitate da stimoli esterni.

I risultati vengono presentati dando spazio dapprima alle voci dei partecipanti dei laboratori riflessivi. Rispetto ad alcuni aspetti ivi trat- 
teggiati, le voci delle intervistate offrono l'opportunità di un approfondimento e vengono dunque presentati in seconda battuta. Per ciascuna cerchia è stato possibile esplorare tanto gli aiuti rivolti ai bisogni concreti di bambini e famiglie (abitazione, vestiti, ricerca di lavoro, attività didattiche ed extradidattiche, accudimento ecc.) quanto quelli rivolti ai loro bisogni emotivi e relazionali. Non si tratta evidentemente di due categorie nettamente distinte, poiché attraverso i primi transitano anche supporti meno tangibili e concreti, ma ugualmente rilevanti per accrescere il benessere di bambini e famiglie in situazione di vulnerabilità.

Tuttavia, nell'analizzare le testimonianze raccolte vi è l'intenzione di porre l'attenzione su entrambe le dinamiche di aiuto per trarne indicazioni diversificate. La prima cerchia è quella più prossima alla famiglia stessa, la propria rete parentale. La seconda investe la sfera amicale, la terza riguarda le relazioni tra genitori e famiglie che frequentano i medesimi contesti formali (educativi, sportivi, parrocchiali), mentre la quarta rappresentata le relazioni che si costruiscono frequentando contesti informali.

La propria rete familiare è facilmente riconoscibile da tutti come il primo riferimento per la costruzione di una vicinanza solidale: «più le persone disponibili alla vicinanza solidale sono ricercate all'interno del contesto naturale di vita della famiglia, più sarà semplice trovarle e più il loro aiuto sarà accettato e riconosciuto come utile dalla famiglia stessa» (Tuggia, 2017, p. 82). A quali bisogni, quindi, rispondono le risorse riscontrabili nei contesti di vita delle famiglie? La propria cerchia familiare rappresenta un riferimento solido per chi si trova a vivere momenti di temporanea o prolungata necessità, in particolar modo per quanto concerne la cura dei bambini. Il supporto offerto dai parenti sembra caratterizzarsi in prima battuta come risposta ai bisogni concreti, mentre solo in seconda battuta vengono menzionati quelli di carattere emotivo e relazionale. «Quello familiare è un supporto concreto e pratico, ma anche emotivo» (Lab1, F2); «i parenti, i nonni, la cerchia della parentela danno una grande mano: accompagnano i figli, li aspettano quando ritornano da scuola ecc.» (Lab2, F3).

Più in generale, nella rete costituita dalla famiglia allargata il confine tra le due tipologie di risposte ai bisogni sembra più difficile da definire, poiché spesso l'una è intrecciata all'altra e all'interno della medesima relazione si valorizzano entrambe le dimensioni.

La seconda cerchia esplorata è quella amicale. La rete è considerata fondamentale soprattutto se gli amici hanno figli delle stesse età o di età simili: questo elemento non è secondario, in quanto le esperienze, le esi- 
genze e le difficoltà legate alla cura dei bambini sono le medesime e spingono le famiglie ad affrontare insieme questi momenti. Spesso amicizia e vicinato tendono a coincidere, e questo facilita molto lo scambio di aiuti e favori e il consolidarsi di supporti pratici bidirezionali e difficilmente quantificabili: «nel condominio dove viviamo ci sono dei vicini di casa con cui ci scambiamo favori e aiuti. La vicina fa da baby-sitter quando noi non ci siamo» (Lab2, F7).

L'abitazione è una risorsa rilevante perché in grado di fornire una risposta concreta alle esigenze delle famiglie. La relazione con i vicini, laddove assume le forme di amicizia o di rapporto basato sulla fiducia, è fondamentale per alleggerire le famiglie di alcuni compiti e far trascorrere ai bambini del tempo insieme.

La risposta ai bisogni concreti sembra essere ulteriormente facilitata nel caso di contesti di ridotte dimensioni demografiche, in cui la prossimità può contribuire a creare dinamiche relazionali solidali e ad alimentare fiducia tra le famiglie. In ciascuno dei tre laboratori riflessivi è stato ribadito che la condizione di genitori, e quindi la presenza di bambini, facilita la creazione di occasioni di vicinanza solidale, che vanno a rispondere anche a bisogni di tipo relazionale, spingendo le famiglie a partecipare alla vita della propria comunità, del proprio paese, a costruire occasioni di incontro e a trascorrere del tempo insieme. «Quando è nata la bambina allora siamo entrati nel contesto sociale del paese. I figli sono un collante che ti permette di costruire reti. L'oratorio lo frequenti se hai figli. Con o senza figli è una differenza abissale» (Lab2, F2).

La rete amicale non veicola solamente aiuti ai bisogni di tipo materiale: è attraverso il dialogo con amici e vicini che si scambiano riflessioni, esprimono dubbi o ricevono consigli e supporto morale utili per il proprio ruolo di genitori. «Con le amicizie c'è anche supporto morale, ci si scambia idee, si parla di perplessità su quello che può essere, quello che la vita ti porta a incontrare» (Lab2, F2).

Questi aspetti emergono anche da alcune interviste individuali, dalle quali si evince come relazioni sorte spontaneamente (di vicinato) o attivate dai servizi sociali, non solo nella cornice di P.I.P.P.I., si trasformino e consolidino in rapporti d'amicizia capaci di rispondere a una molteplicità di tipologie di bisogni, con differenti forme, intensità e tempi.

Ho trovato dei vicini bravissimi alle case popolari, che mi danno una mano. Quasi nessuno ha le famiglie vicine, quindi ci aiutiamo insieme. Domenica mangiamo insieme con i vicini, ognuno cucina qualcosa. Oppure quando si blocca il termostato viene il figlio della vicina ad aggiustarlo. È una rete che si è creata da 
sola, senza i servizi. C'è una signora in particolare che si è attivata e mi ha chiesto se avevo bisogno di aiuto; io ero un po' più chiusa, ma grazie a lei mi sono aperta di più. Mi permette di sfogarmi con lei, e lei si sfoga con me. Grazie ai miei vicini mi sento meno sola. Mi sento più sicura, mi posso fidare di loro (I2).

In questa citazione si ritrovano molti elementi tipici delle relazioni di sostegno informale, come lo spontaneismo, la solidarietà tra vicini, la condivisione di momenti della vita quotidiana, lo scambio di favori e il supporto morale reciproco. Un'altra intervistata sottolinea come la relazione d'aiuto sia proseguita anche dopo la fase più acuta di bisogno, proprio perché maturata in un rapporto di amicizia.

P. c'è ancora, ora ha quattro nipotini e ha meno tempo per me, però ci sentiamo al telefono. Se la chiamo e ho bisogno di lei è sempre presente. [...] adesso con P. restiamo in contatto, è raro qui incontrare una persona così. È nata una amicizia con lei. Per me ha fatto di tutto e di più. [...] Un po' una mamma, me la sento. Ha la capacità di stare calma, quella razionalità sempre sobria, che mi fa ragionare (I4).

La terza cerchia è quella relativa alle relazioni instauratesi tra genitori e famiglie in contesti formali, spesso a partire dalle comuni frequentazioni dei figli (scuola, attività extra scolastiche, parrocchie). Frequentare l'asilo, la scuola, l'oratorio o le attività sportive ha ricadute importanti non solo per i bambini stessi, ma anche per i genitori. In queste occasioni ricorrenti le possibilità per incontrarsi, discutere e scambiare i punti di vista sono più abituali e la condivisione della medesima esperienza alimenta e stimola maggiormente le relazioni.

Quando si frequentano amici che hanno figli coetanei diventa più facile riuscire a gestirli. Nel mio palazzo ora c'è una famiglia con un bimbo della stessa età. L'aiuto solidale è reciproco, io li porto a scuola, tu li vai a prendere. La vicinanza fisica con famiglie con bambini della stessa età è proprio forte (Lab3, F4).

Dentro queste reti si creano, quindi, occasioni di vicinanza solidale che rispondono a volte a bisogni di tipo relazionale, altre volte di tipo più concreto e pratico, come l'accompagnamento, i compiti, l'accudimento temporaneo: «La rete con i genitori dei compagni di scuola dei figli è utile per scambiare qualche idea o per aiutarsi, prendi tu, prendo io... Ad esempio se devono andare a catechismo ci si organizza» (Lab2, F4). «Gli aiuti sono arrivati in maniera amichevole, spontanea: "hai bisogno? Ti porto io i bambini al judo!". Sono aiuti di tipo pratico, concreto. 
C'è la mamma di un bambino che mi aiuta tanto, va a judo, ritira anche i miei due. Il sabato c'è un'altra mamma: "vengo a prenderli...Li porto a calcio"» (I6).

A volte sono i genitori stessi ad aumentare le opportunità di incontro organizzando fra loro momenti conviviali o ricreativi da trascorrere insieme. Questi momenti vedono i genitori investiti di differenti impegni, in base alla propria disponibilità di tempo. In tali reti, che potremmo definire funzionali perché sorte in prima battuta per dare risposta a bisogni concreti come quelli esemplificati in precedenza, possono anche generarsi amicizie che in alcuni casi continuano dopo l'esperienza scolastica e assolvono altre funzioni, assicurando risposte ai bisogni di tipo relazionale ed emotivo.

L'accompagnamento e l'uscita da scuola rappresentano occasioni molto importanti per i genitori perché utilizzano questi momenti per confrontarsi con altri genitori e, in alcune occasioni, per chiedere o offrire un supporto: «Quando aspetti i bambini fuori dalla scuola magari ti confronti» (Lab3, F2).

Anche le attività sportive diventano contesti in grado di creare relazioni, secondo alcuni genitori anche in maniera più immediata e facile rispetto al contesto della scuola: «Lo sport crea comunità. I genitori cucinano per la squadra, diventano una grande famiglia. Lì si creano dei legami anche più facilmente che a scuola. La scuola ha i suoi tempi» (Lab1, F1). «La rete dei genitori del basket è bella, anche per passare momenti piacevoli insieme» (Lab2, F6).

Tra i contesti formali menzionati dai partecipanti ai laboratori riflessivi e dagli intervistati, l'oratorio è quello in cui appare più evidente la potenzialità delle reti sociali in risposta ai bisogni relazionali ed emotivi. L'oratorio è considerato un luogo aperto e sicuro che offre molteplici funzioni: momenti ricreativi ai bambini; occasioni di incontro per i genitori i cui figli lo frequentano; percorsi di confronto e dialogo per famiglie e genitori. Nel panorama delle realtà territoriali extrascolastiche gli oratori garantiscono una presenza capillare e continua e rappresentano una risorsa all'interno della quale utilizzare servizi o organizzarne di nuovi. Dalle interviste è infatti emerso che per alcuni gruppi di persone la parrocchia è un ambiente dentro al quale entrare in contatto con chi condivide gli stessi valori, inserirsi in attività solidali già avviate (doposcuola, raccolta viveri ecc.) e poterne promuovere di nuove. «Ho conosciuto alcune famiglie in parrocchia, e poi da lì abbiamo iniziato a interessarci ad alcuni bambini che vedevamo in situazione di bisogno» (I1). «Io facevo volontariato in parrocchia e poi anche a casa, mi piace 
poter fare qualcosa, anche di pratico. Ho scelto di fare. Ho disponibilità di tempo» (I7).

Dell'oratorio viene più volte sottolineato l'aspetto di apertura verso tutte le fasce sociali e le differenti fedi religiose, mettendone in evidenza la funzione di coesione sociale e integrazione tra famiglie radicate nel territorio e famiglie appena giunte, con bisogni concreti ma soprattutto relazionali.

L'oratorio è un posto dove vengono tutti, aperto a tutti, anziani, bambini, giovani coppie, arriva quasi tutto il paese indipendentemente dalla fede religiosa. All'oratorio vengono anche bambini stranieri, si creano amicizie con le famiglie dei bambini, tutti ti conoscono, anche questa è diventata famiglia, fai da mediatore tra le nuove famiglie arrivate e quelle vecchie (Lab1, F1).

L'oratorio è menzionato anche come contesto di apprendimento, che offre occasioni formative per mettersi alla prova e per iniziare percorsi caratterizzati dalla solidarietà: «All'oratorio ci si incontra per stare bene insieme, per fare un cammino educativo, ma anche per fare cose insieme agli altri e per gli altri [...]. Abbiamo trovato persone che sono venute ad aiutare i nostri figli per qualche materia» (Lab1, F7).

È quindi un luogo dove trovare e ricevere supporti concreti, ma è anche un contesto dove alcune famiglie trovano aiuti di tipo emotivo, punti di riferimento utili per i percorsi di crescita o anche per il semplice stare insieme.

La quarta cerchia, infine, prende in considerazione le relazioni che si costruiscono frequentando contesti informali. I parchi gioco, in città o in paese, vicini alle scuole o ai centri commerciali, sono spesso contesti dove i genitori - specialmente le mamme - trovano un sostegno che potremmo definire "leggero", attraverso il quale scambiare informazioni, ricevere consigli e, in caso di bisogni specifici, chiedere ulteriore supporto. «Le mamme si incontrano, utilizzano il parco giochi per fare incontrare i loro bambini, per uscire dalla solitudine» (Lab2, F5). «Andavo al parco giochi del McDonald's e facevo molte chiacchierate con altre mamme e i bambini giocavano» (Lab1, F2).

Dei luoghi come il Consultorio Familiare o gli ambulatori pediatrici vengono segnalati gli aspetti più informali come le sale d'attesa, che diventano funzionali in chiave di creazione di reti o di relazioni informali d'aiuto, grazie alle occasioni di incontro che offrono. «Il Consultorio è stato d'aiuto, in sala d'attesa c'erano altre mamme che vivevano quelle fatiche, era uno spazio che vivevo come una risorsa d'appoggio, uno spazio di relazione» (Lab3, F5). 
Si registrano diverse pratiche di vicinanza solidale in cui i genitori organizzano "dal basso" occasioni di sostegno reciproco, in risposta tanto a bisogni materiali quanto relazionali. Rispetto ai primi si fa riferimento prioritariamente ad attività da svolgersi nel tempo libero con $\mathrm{i}$ figli (escursioni, feste), in cui alcuni genitori con maggiori disponibilità di tempo si prendono la responsabilità di gestire questi momenti rivolti ai bambini e altri genitori possono beneficiarne sentendosi sollevati da alcune mansioni e semplificando la propria organizzazione quotidiana o settimanale: «d'estate avendo tempo organizzo gite e pomeriggi con bambini per sollevare i genitori degli altri bambini che lavorano» (Lab1, F1).

Queste reti, ovviamente, rispondono anche a bisogni relazionali, creando spazi e momenti dedicati al dialogo e al confronto. Da alcune di queste esperienze sono nate associazioni, o comunque gruppi informali alimentati dai genitori stessi e in grado di attivarne altri e includere anche famiglie in situazione di vulnerabilità. La mobilitazione da parte dei genitori spinge all'interazione e integrazione con altre realtà del territorio, come associazioni, Pro loco, parrocchie. Specialmente in contesti di piccole dimensioni senza una particolare dotazione di servizi per le famiglie e per i bambini sembrano essere di fondamentale importanza le reti spontanee che riescono a organizzare l'aiuto e renderlo fruibile ad altri.

Mi ero rivolta alla Pro loco per avere un locale dove trovarci come genitori, avevamo creato questo spazio, gestito da noi genitori. È servito per socializzare, grazie al fatto di avere figli mi sono inserita, senza figli avrei avuto più difficoltà a entrare nella vita sociale del paese. L'associazione crea appartenenza, qualcuno che vive le tue stesse esperienze in un dato momento (Lab1, F3).

In altri casi la creazione di una rete di supporto, pur nascendo dal basso, può beneficiare di un sostegno economico o di un investimento iniziale da parte di un soggetto terzo. Questo aspetto può rappresentare un input fondamentale per promuovere e consolidare queste esperienze.

Qualche anno fa grazie al progetto XY, si è creata una rete tra genitori dell'asilo. Poi si è creata una rete per realizzare un piccolo campo estivo e si sono messe in gioco mamme che avevano tempo e non sapevano dove portare i figli d'estate e mamme che non avevano tempo. Grazie a questi soldi in più abbiamo sgravato alcune mamme dal dover seguire sempre i figli. E questo è stato molto arricchente, ha creato anche una rete. C'è ad esempio una mamma che non manda i figli a scuola. Grazie a questa iniziativa ha creato dei legami e il gruppo la sta aiutando a capire di cosa ha bisogno (Lab2; F4). 
Le testimonianze raccolte non mancano di menzionare, tra i contesti in grado di fornire risposte ai bisogni delle famiglie, i servizi più formalizzati e strutturati come la scuola, i presidi sanitari, i servizi sociali e quelli specialistici. In assenza di sostegni da parte dei natural helpers (Folgheraiter, Cappelletti, 2011), ad esempio, la scuola sembra essere per alcuni genitori un luogo all'interno del quale ricercare un rapporto privilegiato con gli insegnanti al fine di affrontare le criticità con i propri figli, segnalarle e instaurare relazioni di fiducia. Tali aspettative sono spesso disattese e le difficoltà nelle relazioni scuola-famiglia paiono essere principalmente - ma non solo - di natura comunicativa, rendendo complesso il dialogo, le possibilità di confronto o le eventuali segnalazioni di situazioni critiche:

a scuola si fa fatica a costruire una relazione diretta con gli insegnanti, c'è diffidenza tra insegnante e genitore, l'insegnante ha paura di dirti le cose. Non c'è una conoscenza e non sai come reagirà l'altro, quindi gli insegnanti si fanno problemi a dirti (Lab2, F7).

Per quanto riguarda, invece, i servizi sociali, le riflessioni relative al loro ruolo nell'attivazione della vicinanza solidale tendono a focalizzarsi su tre dimensioni fra loro intrecciate.

La prima riguarda la funzione di connessione tra famiglie in situazione di vulnerabilità e risorse di aiuto che viene idealmente riconosciuta ai servizi. Si tratta di un ruolo di "ponte", ovvero di facilitazione dell'incontro tra chi è disponibile a fornire informalmente risposte ai bisogni materiali o relazionali e chi si trova, appunto, in temporanea condizione di bisogno. Questo ruolo viene riconosciuto anche ad altre realtà come le associazioni o la parrocchia, che indirizzano i genitori verso le risorse d'aiuto disponibili sul territorio.

Quello che ha aiutato, nel nostro caso, è stata la circolazione di informazioni. L'unico modo per creare il ponte è uscire dallo standard istituzionale, la scuola da sola, i servizi sociali da soli ecc. Occorre travalicare il confine. Nel nostro caso è stata l'insegnante di religione che ha messo insieme le risorse e i vari soggetti (Lab3, F2).

Tuttavia, sono pochi i riferimenti ai servizi sociali come attori in grado di creare realmente queste connessioni e assumere, almeno in una fase iniziale, la regia di queste relazioni:

l'ideale sarebbe che ci fosse una rete di amicizia tra famiglie, ma il ruolo formale di qualcuno ci deve essere per individuare le famiglie da sostenere (Lab1, F4). 
La seconda dimensione, emersa in misura maggiore dalle interviste, riguarda le modalità con cui queste connessioni sono facilitate e realizzate, ovvero quali e quante informazioni sulle famiglie in situazione di vulnerabilità i servizi condividono con chi offre il proprio aiuto. Dalle riflessioni raccolte tra chi ha fornito una risposta ai bisogni di bambini e famiglie emerge l'esigenza di ricevere maggiori informazioni dai servizi rispetto alle condizioni e ai livelli di benessere di queste famiglie:

Io vorrei saperne un po' di più sul bambino che aiuto e sulla sua famiglia, dico "ma non è possibile per voi andare a vedere come è la sua giornata?". Se poi le assistenti sociali mi dicono "ok, va bene, tienilo tu", o "lascialo andare a casa" sono più tranquilla. [...] Ma vorrei sapere un po' di più come devo muovermi, vorrei sapere che lui stia bene. [...] Bisognerebbe avere più informazioni sulle famiglie che si aiutano, se non fossero stati gli insegnanti a dirmi qualcosa, io queste cose qua non me le immaginavo nemmeno (I1).

L'apparente disorientamento di alcune figure di supporto è infatti determinato da un accompagnamento che viene percepito come insufficiente in relazione al proprio operato e dalla sensazione di potere/volere ampliare il raggio d'azione della propria relazione d'aiuto. La - almeno per alcune situazioni - scarsa integrazione con il servizio sociale rende incerto il percorso di aiuto e il perimetro dentro al quale mobilitare le risorse.

La gente mi chiede "ma come vivono?". Ma io non lo so e per la parte che devo fare non mi interessa. Però non so...Anche il ragazzo disabile si potrebbe portarlo un po' fuori. Però non so...Bisognerebbe fare un discorso ben più ampio magari con l'assistente sociale. Però a me hanno chiesto questo e per ora faccio così. Avrebbero bisogno come famiglia di qualcosa in più, forse, secondo me...[...]. Non so molto, però l'assistente sociale non mi ha coinvolto in questa cosa (I5).

A fronte di questi mancati coordinamenti, in alcune relazioni d'aiuto le decisioni che riguardano il come e il cosa fare nel corso dell'esperienza vengono assunte direttamente dalle figure di supporto.

L'ampliamento e spostamento delle attività l'ho fatto e deciso tutto io. Mi stupisco che non mi abbia più contattato nessuno per sapere un po' come andava. [...] Non ho un giudizio molto positivo sui servizi sociali, non sono molto presenti, se riescono a lasciare agli altri lo fanno. Invece sono loro che dovrebbero interessarsi, ma forse non hanno il tempo (I5). 
La terza dimensione riguarda la funzione di orientamento e formazione che alcuni intervistati si aspettano dai servizi. Più o meno implicitamente, infatti, emerge non solo l'aspettativa che siano gli operatori sociali a intercedere tra chi aiuta e chi riceve aiuto, ma che spetti a loro il compito di orientare e supportare chi mette a disposizione il proprio aiuto. Durante i laboratori riflessivi è stata più volte sottolineata la necessità, in casi di particolare fragilità, di accompagnare chi offre un sostegno al fine di indirizzare la relazione verso modalità più rispondenti ai bisogni delle famiglie. Ciò rientra, più in generale, nel tema riassumibile dalla domanda "chi aiuta chi offre aiuto?" e attorno al quale i partecipanti hanno evocato l'importanza di un soggetto con un ruolo sia di raccordo (tra famiglie, tra famiglie e territorio, tra istituzioni) sia di sostegno, formazione e orientamento.

Non si può costruire una rete di volontariato senza delle informazioni, una preparazione culturale. [...] Devono essere i servizi sociali che ti devono aiutare a capire come ti devi muovere. [...] È importante in questo senso la formazione, i genitori dovrebbero avere delle competenze e saper accogliere il diverso (Lab3, F1).

I genitori che offrono un aiuto avrebbero bisogno di maggiori strumenti informativi e formativi per capire quello che è possibile fare con l'altro. L'istituzione dovrebbe occuparsi di chi è fragile ma anche di chi vuole offrire supporto. Se non hai strumenti per aiutare rischi di rimanere invischiato anche tu che vuoi dare l'aiuto. Ci vogliono sempre delle competenze, in qualsiasi ambito (Lab2, F1).

Anche le interviste individuali confermano questa esigenza e, in più di un'occasione, il confronto con i servizi sociali ha avuto luogo solo in virtù della pregressa conoscenza tra chi offre il sostegno e il singolo professionista:

ogni tanto chiedo all'assistente sociale come comportarmi con la signora, se alle volte è insistente, o cose così...Conoscendo l'assistente sociale mi sento di chiedere a lei, se non la conoscessi non andrei a disturbare i servizi sociali per queste cose. Quando la ragazzina aveva avuto gli attacchi di panico mi sono confrontata con lei (I7).

Alcuni partecipanti, infine, si sono soffermati sul rischio di compromettere le proprie relazioni familiari a fronte di eccessivi coinvolgimenti personali nelle relazioni d'aiuto. Viene, anche in questi casi, segnalata 
la necessità di essere supportati nel gestire e motivare le proprie scelte all'interno del proprio nucleo familiare.

Mi sto accorgendo che ho paura, nel muovermi, di essere giudicata. Certe cose le faccio (ad esempio compro i biscotti alla bambina che aiuto), ma ho paura che i miei figli siano gelosi. In questo avrei bisogno di essere supportata (Lab3, F3).

Questo aspetto è particolarmente rilevante: la risposta ai bisogni sia materiali che relazionali sembra essere più efficace, puntuale e inclusiva se non è solamente un membro della famiglia a investire sulla relazione d'aiuto, bensì l'intero nucleo. Tuttavia, come sostiene un'intervistata, la famiglia deve essere preparata a questa esperienza e deve trovarsi d'accordo sul perimetro d'azione entro il quale si intende stare.

La persona che accoglie deve parlarne con gli altri componenti della famiglia, deve discuterne. Ad esempio io ho chiarito a mio marito che l'aiuto che avrei dato alla bambina non sarebbe sfociato in un affido. Se c'è una chiusura da parte di alcuni componenti della famiglia la cosa non funziona. Devi preparare il terreno all'interno della famiglia, è fondamentale. Poi gradualmente gli aiuti si ampliano, ma non puoi portare un estraneo dentro senza prepararli prima. Gli equilibri della famiglia vanno rispettati e non stravolti (I3).

\section{Considerazioni conclusive e questioni aperte}

Lo spazio collaborativo tra ricercatori e professionisti nell'approfondimento di Sondrio relativo al tema della vicinanza solidale ha riguardato tutto il percorso della ricerca, dalla fase di costruzione della domanda e dell'oggetto di indagine, al progressivo affinamento del disegno di ricerca, fino allo sviluppo dei processi interpretativi basati sui risultati dei laboratori. In questo tempo sono state rese esplicite letture e interpretazioni diverse rispetto alla vicinanza solidale, che consentono una decostruzione riflessiva delle comprensioni sul tema e che richiedono di intraprendere percorsi di ricostruzione concettuale delle ragioni sottese a tale pratica (Bove, Sità, 2016), di cui in questo paragrafo conclusivo si inizia a dare conto.

La definizione di vicinanza solidale presente nelle Linee di indirizzo (Ministero del Lavoro e delle Politiche Sociali, 2017) fa riferimento al «sostegno alla famiglia nella vita quotidiana, a partire da bisogni circoscritti e concreti, individuati insieme alla famiglia e esplicitamente 
indicati nel progetto» (ivi, p. 71), relativamente allo svolgimento con il bambino di alcune attività e compiti, all'accompagnamento dei genitori nel fronteggiare i problemi della quotidianità e alla promozione dell'integrazione della famiglia nella vita sociale del territorio.

I laboratori riflessivi svolti a Sondrio, a partire da una riflessione sui luoghi e sulle persone cui ciascun partecipante si rivolge per "ricevere aiuto", hanno consentito la descrizione di una molteplicità di vissuti di vicinanza solidale. Particolare evidenza assume una più sfaccettata lettura dei bisogni cui essa può rispondere. Certo, nelle interviste e nei laboratori si fa ampio riferimento ai bisogni concreti definiti nelle Linee di indirizzo, ma a questi si affiancano i bisogni di tipo emotivo/morale, che riguardano il condividere interessi, ricevere consigli, confrontarsi su problematiche comuni, ma anche il semplice stare o fare delle cose insieme. La modalità con cui è intesa la vicinanza solidale si completa dunque: vicinanza solidale è l'aiuto concreto da famiglia (o persona) a famiglia, ma anche l'appartenenza e/o la frequentazione di contesti di gruppo anche informale.

Nel primo caso sono riconoscibili tutti gli aiuti di tipo concreto e/o emotivo che le persone ricevono dai propri familiari e parenti e dai propri amici o conoscenti, nonché dai servizi sociali, dalla scuola, dalle associazioni ecc.

La seconda modalità di vicinanza solidale, invece, riguarda situazioni in cui i genitori si trovano a frequentare altri genitori in ragione della condivisione della frequentazione di spazi comuni, come possono essere la classe scolastica dei figli o l'attività sportiva o associativa (compreso l'oratorio). Sono state citate come occasione di vicinanza solidale la costruzione di incontri informali di gruppo che avvengono nel tempo libero, quando si portano i figli a giocare al parco, per esempio, o nelle sale d'attesa dei consultori familiari. In queste situazioni, l'occasione di incontro è del tutto informale e a volte anche sporadica, ma offre comunque la possibilità di scambiare idee, suggerimenti, informazioni, anche in ragione della stessa condivisione dell'età dei figli.

Ma la possibilità di vivere la vicinanza solidale è riconosciuta anche in altre situazioni informali, come la partecipazione alle feste e alle sagre del paese. In tutte queste situazioni non c'è un bisogno che trova risposta con un aiuto concreto, ma un bisogno emotivo e relazionale, che riguarda anche semplicemente la voglia di stare bene insieme e passare del tempo piacevole in compagnia. È possibile che da queste situazioni informali si sviluppino vere e proprie relazioni di risposta a bisogni concreti, ma non è un tratto necessariamente caratteristico di tali frequentazioni. 
Queste considerazioni conducono a una possibile rivisitazione della definizione di vicinanza solidale così come formulata nelle Linee di indirizzo, con una apertura a interventi in grado di curare la partecipazione delle persone a "occasioni dove stare bene insieme ad altre persone". Queste stesse occasioni potrebbero essere intese come punti di accesso per costruire relazioni in grado di rispondere ai bisogni concreti indicati nelle Linee di indirizzo. In ogni caso, offrendo l'opportunità di relazioni informali vissute positivamente, esse possono contribuire a modificare i pattern interpersonali consueti, fino a un miglioramento della qualità delle relazioni nell'intero sistema familiare (Byng Hall, 1988, trad. it. 1998; Tuggia, Zanon, 2017).

I partecipanti hanno poi evidenziato la necessità di una regia che faciliti la creazione di relazioni di vicinanza solidale e fornisca risposte ai bisogni delle famiglie più vulnerabili. Questa regia non sempre è stata riconosciuta a un soggetto specifico. Le esperienze dei partecipanti a volte rimandano a una regia non costruita appositamente, che è avvenuta quasi involontariamente grazie alla sensibilità di alcune persone fortemente radicate nel territorio, come l'insegnante di religione o la coordinatrice del doposcuola. Incuriosisce come raramente tale regia sia attribuita ai servizi sociali, anzi, talvolta è esplicitamente negata a questi ultimi, che appaiono percepiti come distanti. Gli operatori invece sembrano rivendicare questo ruolo. Quando invece i partecipanti si riferiscono a relazioni di vicinanza solidale già instaurate, al servizio sociale è attribuito un compito forte di cura e di accompagnamento, che non sempre trova realizzazione nelle esperienze considerate.

La rivendicazione del ruolo di regia sul territorio da parte degli operatori del servizio sociale, il contestuale non riconoscimento di tale compito da parte dei partecipanti ai laboratori riflessivi e, dall'altra parte, il fatto che gli stessi partecipanti individuino nel servizio la competenza per rispondere alle domande che il vivere relazioni di vicinanza solidale genera, porta a interrogarsi sui compiti e sulle modalità in cui il servizio sociale interpreta la gestione e la promozione della vicinanza solidale. Sembra che tra i partecipanti sia presente l'idea che il servizio sociale sia necessario nel momento in cui si fa riferimento alla singola relazione d'aiuto, con una richiesta prestazionale di garantire informazioni e supporto. La stessa presenza non appare necessaria quando ci si colloca nel territorio e nel tessuto di relazioni naturali, cui pure la vicinanza solidale fa riferimento.

La presenza del servizio sociale in relazione alla vicinanza solidale sembra «riferibile solo alle difficoltà dei singoli da sostenere nel pro- 
cesso di integrazione in una comunità» (Allegri, 2015, p. 19) e non a un lavoro «nel territorio con i diversi attori che ne fanno parte» (Ibidem). Rafforzare la visibilità e la presenza del servizio sociale nel territorio potrebbe, invece, essere la via per rispondere alle difficoltà di attivazione del dispositivo della vicinanza solidale, più volte evidenziate sia dagli operatori di Sondrio che nei risultati del Programma P.I.P.P.I. Come ben espresso da Allegri:

le amministrazioni pubbliche sono tradizionalmente abituate a pensare che gli utenti arrivino ai loro uffici per presentare domande e in tal senso hanno costruito strutture, servizi e processi di risposta. Tuttavia [...] le persone più in difficoltà $[. .$.$] non si presentano spontaneamente. Non solo, questo atteggia-$ mento riguarda anche molti cittadini, delusi e sfiduciati rispetto alla relazione con le istituzioni (ivi, p. 104).

L'approfondimento sulla vicinanza solidale portato avanti nei laboratori riflessivi di Sondrio porta a considerare un cambiamento di prospettiva che propone di procedere in senso opposto: uscire dai servizi e andare a cercare le persone, stare nei luoghi delle persone per creare intenzionalmente contesti dove si vivono le relazioni. Si tratta di sviluppare nuovi percorsi di collaborazione tra contesti informali e formali, dove una possibile futura linea di azione potrebbe riguardare:

- il ripensamento del ruolo e della posizione del servizio sociale nella regia di occasioni di vicinanza solidale, anche in ragione dell'esigenza di un radicamento territoriale;

- il ripensamento della vicinanza solidale considerando la duplice accezione di (i) aiuto concreto da famiglia (o persona) a famiglia e di (ii) cura dei contesti informali del vivere in relazione, dove le persone possano semplicemente sperimentare di "stare bene insieme" oppure da intendere anche come punto di accesso per la creazione di nuove relazioni sociali di aiuto e sostegno.

Lo spazio collaborativo tra ricercatori e professionisti a Sondrio lascia aperta e da esplorare la questione della costruzione dell'innovazione sociale: il presente studio si ferma un momento prima della riprogettazione delle pratiche di vicinanza solidale, che dovrà necessariamente coinvolgere anche la cittadinanza insieme ai professionisti; esso è una testimonianza di come l'innovazione sociale prima di essere pensata e messa in campo richieda di dedicare tempo all'analisi e all'emersione delle differenti comprensioni da parte dei partecipanti. 


\section{Ringraziamenti}

Gli Autori ringraziano tutti gli operatori e le persone che hanno preso parte al percorso del Laboratorio Territoriale. In particolare si ringraziano Luca Verri, Barbara Negri e Katiuscia Patalano per l'impegno profuso e la determinazione nel portare avanti questa iniziativa di ripensamento critico e innovazione.

\section{Riferimenti bibliografici}

Allegri E. (2015): Il servizio sociale di comunità. Roma: Carocci.

Allegri E., Eve M., Mazzola R., Perino M., Pogliano A. (2020): Other "Lenses": A Training Programme for Social Workers and Others Working with Asylum Seekers and Migrants in Italy. European Journal of Social Work, 23(3), pp. 529-540.

Bondioli A., Ferrari M. (2004): Verso un modello di valutazione formativa. Ragioni, strumenti, percorsi. Bergamo: Edizioni Junior.

Borsellino P., Belotti A. (2010): Manuale Operativo Progetto Famiglie in Rete. Feltre: Ulss 8, (http://www.ulssfeltre.veneto.it/shared/UserFiles/file/ Link\%20Veloci/Mercoledi\%20della\%20salute/Manuale\%20Operativo $\% 20$ Progetto $\% 20$ Famiglie $\% 20$ in $\% 20$ Rete.pdf; data di ultima consultazione: 23.9.20).

Bove C. (2009): Ricerca educativa e formazione. Contaminazioni metodologiche. Milano: FrancoAngeli.

Bove C., Sità C. (2016): Col-legare le voci nella ricerca. Sostenere esperienze di inquiry collaborativa tra ricercatori e professionisti. Encyclopaideia, XX(44), pp. 57-72.

Brown J., Isaacs D. (2005): Shaping Our Future through Conversations that Matter. San Francisco (CA): Berrett-Koehler Publ.

Byng Hall J. (1988): Le trame della famiglia. Trad. it. Milano: Raffaello Cortina, 1998.

Chaskin R.J., Brown P., Venkatesh S.A., Vidal A. (2001): Building Community Capacity. New York: Aldine.

Dewey J. (1938): Logic: The Theory of Inquiry. New York: Henry Holt \& Co.

Di Masi D., Serbati S., Sità C. (2019): La collaborazione tra ricerca e pratiche professionali per l'innovazione sociale: i laboratori territoriali nella prospettiva della Teoria dell'Attività. Studium Educationis, XX(1), pp. 91-112.

Durand D., Massé R., Ouellet F. (1989): Intervenantes non professionnelles et prévention de l'enfance maltraitée, Évaluation du projet de la visite. Santé mentale au Québec, 14(2), pp. 26-38.

Folgheraiter F., Cappelletti P. (a cura di) (2011): Natural helpers: storie di utenti e familiari esperti. Trento: Erickson. 
Guba E., Lincoln Y. (1989): Fourth Generation Evaluation. Newbury Park (CA): Sage.

Hostinar C.E., Sullivan R.M., Gunnar M.R. (2014): Psychobiological Mechanisms Underlying the Social Buffering of the Hypothalamic-Pituitary-Adrenocortical Axis: A Review of Animal Models and Human Studies across Development. Psychological Bulletin, n. 140, pp. 256-282.

Iannone R. (2007): Le cerchie sociali in G. Simmel. Rivista Trimestrale di Scienza dell'Amministrazione, n. 3, pp. 28-39.

LabRIEF (2019a): Struttura di governance e piano di lavoro P.I.P.P.I. 2019-2021. Padova: Laboratorio di Ricerca e Intervento in Educazione Familiare.

LabRIEF (2019b): P.I.P.P.I. Report conclusivo. Sesta implementazione (20172018). Padova: Laboratorio di Ricerca e Intervento in Educazione Familiare.

Lacharité C. (2014): Transforming a Wild World: Helping Children and Families to Address Neglect in the Province of Quebec, Canada. Child Abuse Review, n. 23, pp. 286-296.

Lacharité C., Éthier L., Nolin P. (2006): Vers une théorie écosystématique de la négligence envers les enfants. Bulletin de psychologie, 59(4), pp. 381-394.

Lines D.R. (1987): The Effectiveness of Parent Aides in the Tertiary Prevention of Child Abuse in South Australia. Child Abuse and Neglect, n. 59, pp. 507-512.

Maurizio R., Perotto N., Salvadori G. (2015): L'affiancamento familiare. Orientamenti metodologici. Roma: Carocci.

Milani P. (2006): L'aiuto informale tra famiglie: ragioni ed esperienze. In R. Maurizio, F. Belletti (a cura di): La prossimità tra famiglie. Padova: Fondazione Zancan, pp. 30-59.

Milani P., Ius M., Serbati S., Zanon O., Di Masi D., Tuggia M. (2015): Il Quaderno di PIPPI. Teorie, metodi e strumenti per l'implementazione del programma. Padova: Becco Giallo.

Ministero del Lavoro e delle Politiche Sociali (2017): Linee di Indirizzo nazionali "L'intervento con bambini e famiglie in situazione di vulnerabilità". Roma: Ministero del Lavoro e delle Politiche Sociali.

Natland S. (2015): Dialogical Communication and Empowering Social Work Practice. Journal of Evidence-Informed Social Work, 12(1), pp. 80-91.

Peirce C.S. (1992): Selected Philosophical Writings. Bloomington: Indiana University Press.

Raineri M.L. (2013): Community Care. In A. Campanini (a cura di): Nuovo Dizionario di Servizio Sociale. Roma: Carocci, pp. 146-149.

Serbati S. (2016): Il supporto sociale informale: esperienze e prospettive in P.I.P.P.I. - Programma di Intervento Per Prevenire l'Istituzionalizzazione. RIEF-Rivista Italiana di Educazione Familiare, n. 2, pp. 95-116.

Simmel G. (1910): Forme e giochi di società. Problemi fondamentali della sociologia. Trad. it. Milano: Feltrinelli, 1983.

Taylor S.E. (2011): Social Support: A Review. In H.S. Friedman (a cura di): Oxford Handbook of Health Psychology. New York: Oxford University Press, pp. 189-214. 
Tuggia M. (2006): Dall'affidamento familiare a esperienze di prossimità. In R. Maurizio, F. Belletti (a cura di): Progetti di prossimità tra famiglie. Padova: Fondazione Zancan, pp. 68-74.

Tuggia M. (2017): La vicinanza solidale. RIEF-Rivista Italiana di Educazione Familiare, n. 2, pp. 77-93.

Tuggia M., Zanon O. (2017): La partecipazione della famiglia al suo percorso di accompagnamento nel Programma P.I.P.P.I. Quali competenze per i professionisti dei servizi? RIEF-Rivista Italiana di Educazione Familiare, n. 2, pp. 25-39. 